\title{
A study of undergraduates' understanding of vector - decomposition of forces on inclined planes
}

Dan Liu and Hashini Mohattala

Department of Physics, University of Hartford, 200 Bloomfield Ave., West Hartford, CT 06117

Students often struggle to decompose a vector, especially when calculating the force components of an object on an inclined plane. In this study, we designed an online vector survey in the context of physics and implemented it in a primarily undergraduate university. The study focuses on the students' learning difficulties and teaching strategies associated with vector decomposition on inclined planes. The analysis of the students' responses indicates that translating a vector to the position with geometric convenience can help them identify angles and calculate vector components on inclined planes more accurately; when students are provided with the definition of the angle in the vector component formula, their performance improves.

2021 PERC Proceedings edited by Bennett, Frank, and Vieyra; Peer-reviewed, doi.org/10.1119/perc.2021.pr.Liu Published by the American Association of Physics Teachers under a Creative Commons Attribution 4.0 license. Further distribution must maintain the cover page and attribution to the article's authors. 


\section{INTRODUCTION}

Applying the knowledge of vectors to kinematics and dynamics is an essential skill for solving physics problems at the undergraduate level. Vectors first appear in the calculations of physical quantities such as displacement, velocity, and acceleration and then in the analysis of free body diagrams. The learning difficulties that undergraduates encounter in vectors are an obstacle in their successful pursuit of physics courses.

Decomposition is one of the first lessons that is taught about vectors. It is an essential step in the calculations of two-dimensional motions and the analysis of free body diagrams in applications of Newton's laws. The intensive usage of vector decomposition and its close correlation to undergraduates' success in introductory level, calculusbased physics courses, make understanding it well an absolute necessity.

Various efforts have been made to address students' learning difficulties in vector decomposition, particularly in simple cases. Knight's study in 1995 included two decomposition questions on algebraic and geometric aspects in Vector Knowledge Test and the results indicated that less than half of undergraduates had sufficient vector knowledge before taking a calculus-based physics course [1]. Barniol and Zavela asked students who had finished introductory physics courses three decomposition questions in graphical format in the 20 question Test of Understanding of Vectors. Based on the responses, they developed a taxonomy of the most frequent errors [2]. Our recent investigation revealed that several learning difficulties are due to a lack of understanding of the connections between the algebraic and geometric aspects of vectors [3].

With the framework of physics, vector-related applications become more challenging. Aguirre's investigation of vector kinematics in projectile motion showed that high school students had numerous incorrect preconceptions with components of a velocity vector and half of undergraduates who had completed a course in mechanics maintain their preconceptions and kept their intuitive beliefs [4, 5]. In Mechanics Baseline Test, Hestenes and Wells included kinetic vectors and superposition of force vectors. The lowest scores of test questions came from the ones requiring vector properties [6]. Modifications to physics lecture instruction had been made and implemented based on curricula such as Tutorials in Introductory Physics and Physics by Inquiry, but the improvement of students' performance in questions about forces and Newton's second law was not more than moderately successful [7-9]. The investigations of Flores et al. show that students' correct response rate of vector questions in the context of forces and dynamics is lower than $50 \%$ on average after implementing the modified lecture of Tutorials in Introductory Physics.

Previous studies and our teaching observations show that the majority of students perform worse on force decomposition when an object is placed on an inclined plane than when it is on a flat surface. The study of Mikula and Heckler showed that $50 \%$ of students could calculate the component of the weight parallel to the surface of the inclined plane correctly and only around $10 \%$ of them could correctly calculate the component of the weight perpendicular to the surface of the inclined plane, which is far below the requisite accuracy needed for such fundamental skills [10].

In extension of previous research, we focus on students' learning difficulties behind two major common errors (Sin/Cos error and MultiDivid error) quantitatively. In order to address common errors and a lack of understanding of the connections between the algebraic and geometric aspects of vectors in the context of physics, we also investigate the effectiveness of two methods that we can easily apply to improve students' performance in vector decomposition on inclined planes by using (1) translation of vector arrows (2) restatement of the angle definition in the vector component formula.

\section{STUDY DESIGN}

In three consecutive semesters in the academic year of 2020 and the spring semester of 2021, a survey focusing on vector decomposition on inclined planes was designed and implemented in an undergraduate-based university in the United States. The students taking University Physics at the calculus level were invited to take the survey and 69 students completed and submitted the survey voluntarily (32 students took version $\mathrm{A}$ and 37 did version B) with the motivation of improving their performance in classroom participation.

The survey was designed for online implementation, which is unavoidable during the pandemic period. We chose the format of multiple-choice questions so that it would be convenient for participants to submit their responses. The number of participants was sufficient to give statistically significant results as shown in Section III.

The survey consists of four multiple-choice questions with two versions [11]. All four questions are related to identifying and calculating the Y component (the component perpendicular to the surface of the inclined plane) of the weight of an object on an inclined plane shown in Figure 1. In order to calculate the components of the weight of an object on an inclined plane correctly, students need to master graphical techniques of resolving vectors into components, including realizing the significance of different projection axes (XY coordinates), formulating right-angle triangles, identifying angles and then using trigonometric ratios correctly to compute the components. These skill elements are included and divided to be tested separately.

The difference between the two versions is designed to address the proposed method (1). The weight of an object is marked at the center of mass in the diagrams in Version A while in Version B, it is marked at the Positions 


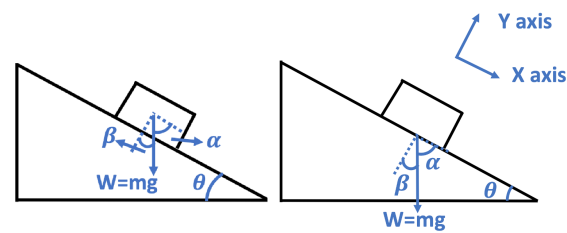

Q1. in Version A and B

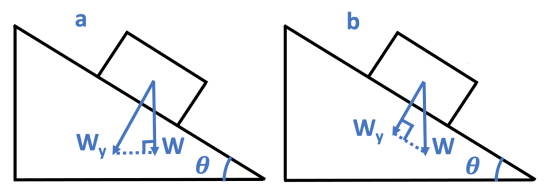

Q2. in Version A

FIG. 1: Free body diagrams in the survey [11]. The upper left two diagrams are for Q1 in Version A and B; the two diagrams at the bottom left are for multiple-choice A and B of Q2 in Version A; the right one is for Q3 and Q4 in Version A. For the diagrams of Q2, Q3 and Q4 in Version B, the vector of weight is marked at the base of the object.

with Geometric Convenience (PGC), which is the base of the object in this case. It is not our intention to obey the typical rules of free body diagrams where forces are attached at the center of mass. The translation of vectors to PGC is applied when students analyzing the diagrams to calculate components. It is an optional tool for students' convenience. Students can take advantage of the fact that vectors keep their directions and magnitudes in translation. Translation of vectors is also inevitable in graphical methods of vector addition and subtraction.

\begin{tabular}{|c|c|c|c|c|}
\hline Common Mistakes & Q1 & Q2 & Q3 & Q4 \\
\hline Correct & $1 \mathrm{~b}$ & $2 \mathrm{~b}$ & $3 \mathrm{f}$ & $4 \mathrm{c}$ \\
Sin/Cos error & & & $3 \mathrm{a}, \mathrm{e}$ & $4 \mathrm{~d}$ \\
Angle error & $1 \mathrm{a}$ & & & $4 \mathrm{~b}$ \\
MultiDivid & & & $3 \mathrm{c}, \mathrm{d}, \mathrm{g}, \mathrm{h}$ & \\
Component & & $2 \mathrm{a}$ & & \\
Sign error & & & $3 \mathrm{a}, \mathrm{b}, \mathrm{c}, \mathrm{d}$ & $4 \mathrm{a}$ \\
CTF & & & & $4 \mathrm{c}, \mathrm{d}$ \\
\hline
\end{tabular}

TABLE I: Categories of the multiple choices for Questions (Q1 - Q4) in the survey: Sin/Cos error, Angle error, MultiDivid error, Component, Sign error and CTF. For example, multiple-choice question Q1 has two choices. 1b is the correct answer and 1a can be selected because of Angle error.

As shown in Table I, the multiple choices of each question are designed and categorized according to the students' common responses in previous studies $[2,10,12]$. Sin/Cos error is that the student switches sine and cosine functions when trying to get a component. Angle error happens when a wrong angle is used in decomposing a vector in the geometric aspect. MultiDivid error is the category of choosing magnitude to divide the trigonometric function to get a component, e.g. $F / \sin (\theta)$ instead of
$F \cdot \sin (\theta)$. Component error is often caused by the lack of understanding of the concept of components of a vector and the inability to identify components in a graph. Sign error occurs when the answer has the wrong sign. The use of Complementary Trigonometric Functions (CTF) e.g. $F \cdot \cos (90-\theta)$ instead of $F \cdot \sin (\theta)$, is not an error. Some choices fall into more than one category.

The above-listed common errors are in two tiers. Sin/Cos error and MultiDivid error are in the upper tier and they can be caused by Angle error, Component error or other learning difficulties. In order to identify them, we utilized intermediate steps of calculating components for Questions 1 and 2 as shown in Figure 1. Question 1 was designed to identify the reason for making Sin/Cos error. Sin/Cos error can come from applying a wrong trigonometric function or having an Angle error. In Question 1, students are asked to select either $\alpha$ or $\beta$ to be equal to $\theta$. Question 2 is intended for MultiDivid error. Students who have difficulty in applying the trigonometric function for opposite/adjacent sides in a right triangle and those who cannot identify components (choice 2a) can end up with the same error. Here, students choose either diagram a or $\mathrm{b}$ for the $\mathrm{Y}$ component of the weight.

Questions 3 and 4 have the same topics but with different choices. They are designed as pairs to test the improvement of implementing the proposed method (2). The study of Mikula and Heckler indicates that a notable proportion of students appear to be confused about when to use sine and cosine functions, instead, they simply use a memorized algorithm using a given angle [10]. In teaching, we also notice that students often question the reason for associating sine angle (the angle of inclination) for the $\mathrm{X}$ component of gravity when the $\mathrm{X}$-axis is aligned with the incline whereas the formula of the $\mathrm{X}$ component is magnitude times cosine angle. The learning difficulty is possibly correlated to the inadequate understanding of the definition of the angle in the component formula.

In Questions 3 and 4, we intend to test whether and how much the restatement of the definition of the angle can help students to find the relevant components. The differences between the two questions are that the definition of the angle in the component formula is given before Question 4 and CTF are available in multiple-choice options of Question 4. The hint of the angle definition helps students to identify the angle in the graph and build a connection between component formula and the geometric aspect of vectors.

\section{DISCUSSION}

In this investigation, our analysis is based on all the submissions, 32 students in Version A and 37 students in Version B. The average score of overall performance is $47 \%$. (It is the same as the average difficulty index as shown in Table II, when all 4 questions are counted equally.) The students who took survey Version A and the ones who took the survey Version B received grades 
of $44 \%$ and $51 \%$ respectively.

\begin{tabular}{|c|c|c|c|c|c|c|}
\hline Index & Q1 & Q2 & Q3 & Q4 & Ave. & Des. Val. \\
\hline$P$ & 0.80 & 0.65 & 0.16 & 0.29 & 0.47 & {$[0.3,0.9]$} \\
$P_{A}$ & 0.72 & 0.69 & 0.13 & 0.22 & 0.44 & {$[0.3,0.9]$} \\
$P_{B}$ & 0.87 & 0.62 & 0.19 & 0.35 & 0.51 & {$[0.3,0.9]$} \\
\hline$D$ & 0.96 & 1.01 & 0.32 & 0.58 & 0.72 & $\geq 0.3$ \\
$r_{p b s}$ & 0.43 & 0.50 & 0.58 & 0.57 & 0.52 & $\geq 0.2$ \\
\hline
\end{tabular}

TABLE II: The average difficulty index of each question in survey Version A and B $(P)$, the ones for Version $\mathrm{A}\left(P_{A}\right)$ and for Version $\mathrm{B}\left(P_{B}\right)$ are listed respectively. Item discriminatory index $(D)$ and point-biserial coefficient $\left(r_{p b s}\right)$ for each question are included. The table also includes the average statistical parameters of all test questions in this study and the desired values (Des. Val.) taken from reference [13].

The validity of the survey is assured by adapting questions in previous studies and common examples in introductory physics courses. The survey was also reviewed by 5 experienced Physics instructors in different institutes, and it was edited based on their feedback. In Table II, we present three types of statistical parameters which commonly applied to evaluate the reliability and discriminatory power of a test [13]. Most evaluation data for the survey questions falls in the desired ranges, but not the difficulty index $P$ in Question 3 and 4 . The difficulty index is calculated by taking the ratio of the number of correct responses on a question to the total number of participants. The higher the difficulty index is, the easier the question is. The lower than desired difficulty index of Question 3 is the reason behind this investigation. As shown in the table, $P_{B}$ in Question 3 increases to 0.19 when the proposed method (1) is implemented and $P_{A}$ in Question 4 increases to 0.22 when the proposed method (2) is implemented. With both proposed methods, the difficulty index reaches 0.35 , falling in the desired range.

The discriminatory index $D$ measures discriminatory power, the extent to how well a single test item can distinguish the students with and without robust knowledge. If many items have high $D$ values in a test, the test is useful in separating strong and weak students. The point-biserial coefficient $r_{p b s}$ is a reliability index, which measures the consistency of a single test item to in the whole test. If an item has a high $r_{p b s}$ value, the students with high total scores are more likely to answer item correctly.

\section{A. Learning difficulties behind common errors}

The accuracy of the first three questions is $80 \%, 65 \%$, and $16 \%$ respectively. Accuracy drops dramatically from Questions 1 and 2 to Question 3. This means that most students can identify the angle $\theta$ and the $\mathrm{Y}$ component of weight successfully, nevertheless, they don't end up with a correct answer for the Y component in Question
3. The following is an analysis of the reasons behind the above large drop in correct response rate. Figure 2c shows Sin/Cos error and Sign error are the two main errors in students' responses. MultiDivid error is minor.
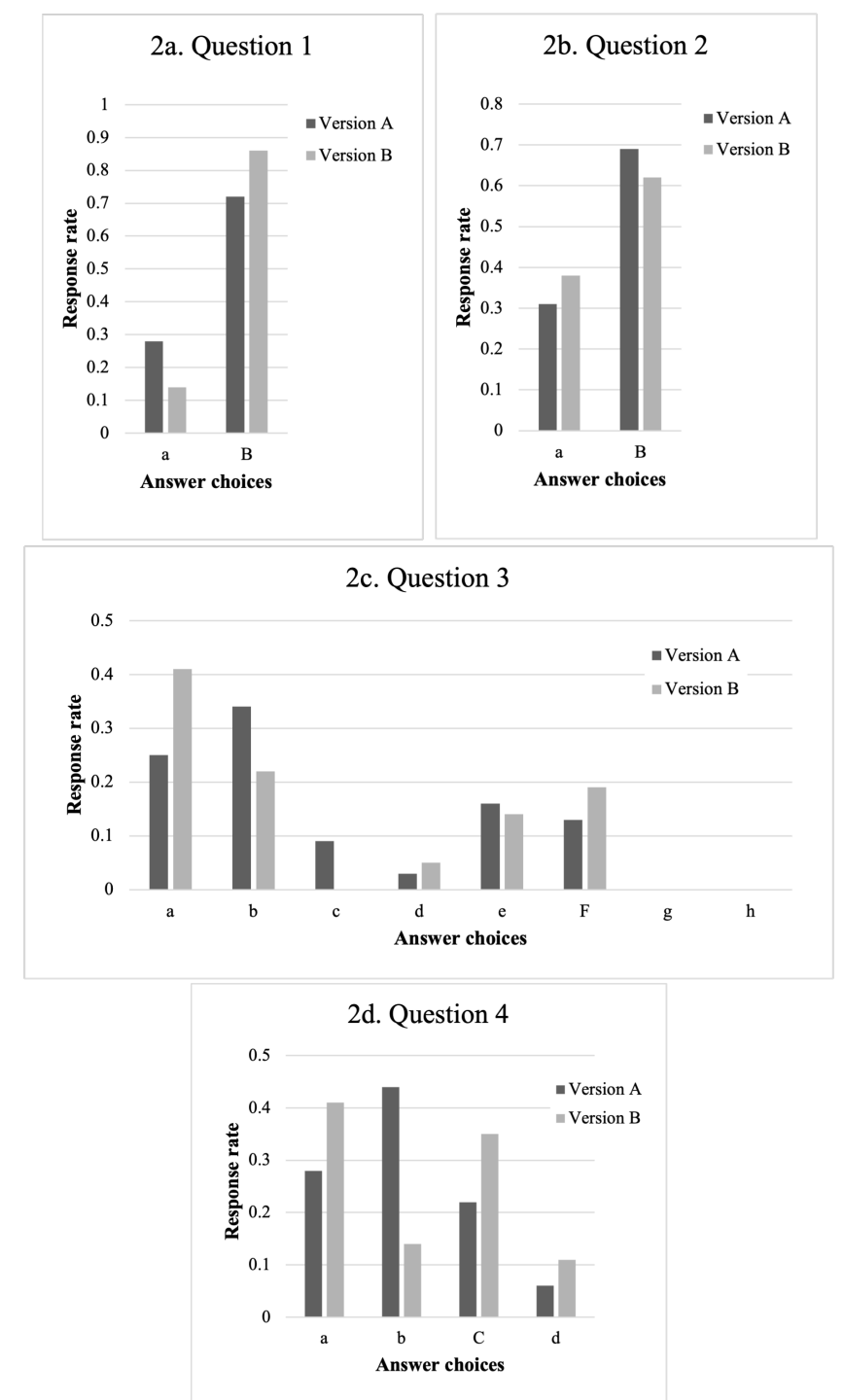

FIG. 2: Students' responses to each multiple-choice option, where the correct option is capitalized. The responses in Version A are in black bars and the ones in Version B are in gray bars.

In Question 3, 48\% of the total participants made Sin/Cos error ( $3 \mathrm{a}$ and e). While going through individual responses, we found that $79 \%$ of these students correctly answered Question 1 of identifying angles correctly. It means that they had reached the stage of trying to calculate the adjacent sides of a triangle, where a cosine function should be applied. This result suggests that approximately $4 / 5$ of the learning difficulties behind students' Sin/Cos error are caused by either not being able to select a correct trigonometric function for the calculation of adjacent sides or failing in reasoning processes.

For MultiDivid error (3c, d, g and h), the survey re- 
sults show that the major learning difficulty is also about trigonometry. $33 \%$ of the students who had MultiDivid errors made mistakes in Question 2. The students who answered both Question 1 and Questions 2 correctly didn't make MultiDivid errors. In other words, nearly $1 / 3$ of the learning difficulties behind MultiDivid error on inclined plane questions are attributable to not being able to identify the $\mathrm{Y}$ component in graphs and the remainder are potentially caused by a lack of knowledge of trigonometry.

As shown in Figure 2a and 2b, Angle error in Question 1 and Component error in Question 2 are minor with comparably high correct response rates, but students' performance on vectors is expected to be improved if more practice in triangle geometry and the emphasis on the concepts of vector components are given in class.

\section{B. Comparison of results with/without applying the proposed methods}

The difference between the two versions is the placement of the vectors at the center of mass or PGC. In Question 2, identifying components is irrelevant to the placement of the weight vector, so the comparison between two versions should not show differences. Consistent with our prediction, $P_{A}$ and $P_{B}$ of Question 2 are nearly the same in Figure 2b. The data also indicates that the students who participated in both versions have a similar level of understanding of vectors.

The proportion of correct responses to Questions 1, 3 and 4 are higher in Version B. The advantage of translating a vector to PGC comes from the fact that students can identify the angles between the vector and its components with greater ease. $87 \%$ of students can identify the angles next to the weight vector in the diagram when the vector arrow is marked at the bottom of the object. In comparison, $72 \%$ answer Question 1 correctly when the weight arrow is marked on the center of mass of the object in Version A. The proportion of correct responses to Question 3 and 4 in Version B is nearly $50 \%$ higher than the ones in Version A. The t-Test of the survey Questions 1, 3 and 4 in Version A and B indicates the improvement statistically significant $(p=0.0321)$. This result shows that the proposed method (1) effectively helps students to decompose a force on an inclined plane.

Not only does it improve students' performance of force decomposition in comparably complex graphical settings, but translating a vector to PGC also helps them to have a better understanding of vector translation in space in general. Nguyen's investigation of vector addition, magnitude and direction in graphical form indicated that students seem to lack the understanding that the magnitude and direction of a vector are strictly preserved by parallel transport [14]. We believe that the method (1) can help students' performance of the calculation of vector addition in the geometric aspect.
The results in Question 4 show that the students' performance improved when the definition of the angle in the component formula is given. The correct response increases from 16\% in Question 3 to $29 \%$ in Question 4. We conducted a t-Test to compare students' performance in Questions 3 and 4 . The result showed a statistically significant improvement $(\mathrm{p}=0.0190)$. A more substantial improvement is expected in teaching practice, by the considerations of our survey design and implementation. First, Question 3 and 4 have the same topic and are next to each other in the survey; the hint of the angle definition before Question 4 may not catch students' attention to rethink and redo the question. The other factor is that students may not be confident with using CTF without much exposure to CTF representations.

With a good understanding of the definition of the angle in the component formula, students' skills at vector decomposition were enhanced with the addition of alternative CTF options which allowed them to avoid Sin/Cos and sign errors. As going through individual responses, we find that $77 \%$ of the students who chose CTF options answered the questions correctly. Sin/Cos error (4d) was greatly reduced to $6 \%$ in Question 4 in Version $\mathrm{A}$ and $11 \%$ in Version B as shown in Figure 2d. Applying the vector component formula directly also avoided the mistake of omitting signs, which is another major common error as shown in our Question 3.

\section{CONCLUSION}

This study shows that the students' learning difficulties behind Sin/Cos and MultiDivid errors in the calculation of force decomposition on inclined planes are trigonometry (major), geometry and concept of components (comparably minor). The results of our investigation also prove that the proposed two methods are effective to improve students' performance, (1) translate vectors to the position with geometric convenience; (2) restate the definition of the angle in vector component formula. The suggested teaching strategies include reviewing vector knowledge along with related trigonometric knowledge before teaching vectors in physics, informing students that they can translate a vector with the full preservation of its magnitude and direction and they can translate forces to the positions with geometric convenience in force decomposition, emphasizing the angle definition and labeling the angle in graphs when introducing vector component formula.

It's imperative to research further on vector related teaching and learning because of the importance of its applications to physics and in the face of students' inadequate understandings of vectors. In the next stage, we plan to include the investigation of Sign error and implement the surveys in the format of open-ended questions so that students' reasoning in the calculation of vectors can be observed and interpreted. 
[1] R. D. Knight, The vector knowledge of beginning physics students, Phys. Teach. 33, 74-78 (1995).

[2] P. Barniol and G. Zavala, Test of understanding of vectors: A reliable multiple-choice vector concept test, Phys. Rev. ST, Phys. Educ. Res. 10, 010121 (2014).

[3] D. Liu and Y. Kottegoda, Disconnect between undergraduates understanding of the algebraic and geometric aspects of vectors, Eur. J. Phys. 40, 035702 (2019).

[4] J. M. Aguirre, Student preconceptions about vector kinematics, Phys. Teach. 26, 212216 (1988).

[5] J. M. Aguirre and G. Rankin, College students' conceptions about vector kinematics, Phys. Educ. 24, 290294 (1989).

[6] D. Hestenes and M. Wells, A mechanics baseline test, Phys. Teach. 33, 159-166 (1995).

[7] P. S. Shaffer and L. C. McDermott, A research-based approach to improving student understanding of the vector nature of kinematical concepts, Am. J. Phys. 73, 921 (2005).

[8] S. Flores, S. E. Kanim and C. H. Kautz, Student use of vectors in introductory mechanics, Am. J. Phys. 72, 460 (2004).

[9] S. Flores-Garca, L. L. Alfaro-Avena and O. Dena-Ornelas $\mathrm{O}$, Students understanding of vectors in the context of forces, Rev. Mex. Fis. E 54, 714 (2008).

[10] B. D. Mikula and A. F. Heckler, Student Difficulties With Trigonometric Vector Components Persist In Multiple Student Populations, 2013 PERC Proceedings.

[11] Vector survey.

[12] J. Van Deventer, Masters thesis, p. 189, (The University of Maine, 2008).

[13] L. Ding, R. Chabay, B. Sherwood, and R. Beichner, Evaluating an electricity and magnetism assessment tool: Brief electricity and magnetism assessment, Phys. Rev. ST, Phys. Educ. Res. 2, 010105 (2006).

[14] N. Nguyen and D. E. Meltzer, Initial understanding of vector concepts among students in introductory physics courses, Am. J. Phys. 71, 630 (2003). 